\title{
Akzidentelle Überdosierungen von Low Dose Methotrexat
}

\author{
Beat Damke ${ }^{a}$, Ruedi Stoller ${ }^{b}$, Margrit Leuthold", David Schwappach ${ }^{d}$ \\ a Dr. med, Clinical Reviewer Swissmedic; ${ }^{b}$ Senior Expert Swissmedic; ${ }^{\circ}$ Dr., Geschäftsführerin Stiftung Patientensicherheit Schweiz; \\ d Prof. Dr., MPH, Wissenschaftlicher Leiter Stiftung Patientensicherheit Schweiz
}

\begin{abstract}
Aufgrund neuer Berichte über schwerwiegende Komplikationen durch tägliche statt wöchentliche Verabreichung von Low Dose Methotrexat bei Patienten mit rheumatoider Arthritis oder Psoriasis erinnern wir an die zwingend erforderlichen Vorsichtsmassnahmen. Fehler entstehen häufig an Schnittstellen, z.B. nach Wechsel der Institution, des Präparats, oder bei Ferienvertretungen. Daher müssen systematische Checks und Kontrollen von der Verschreibung über die Abgabe bis zur Instruktion von Patienten und Angehörigen sicherstellen, dass Low Dose Methotrexat nur $1 \times$ wöchentlich gegeben wird.
\end{abstract}

Swissmedic und Patientensicherheit Schweiz haben bereits 2012 [1] auf schwere Zwischenfälle durch tägliche anstelle wöchentlicher Verabreichung von Low Dose Methotrexat bei Patienten mit Rheumatoider Arthritis und Psoriasis aufmerksam gemacht. Solche Patienten erhalten üblicherweise 10-15 mg wöchentlich; eine tägliche Anwendung dieser Dosierung führt zu schweren Intoxikationen mit teilweise letalem Ausgang.

Gleichzeitig hat die Stiftung für Patientensicherheit in Zusammenarbeit mit verschiedenen Fachexperten und Fachorganisationen einen Quick Alert ${ }^{\circledR}$ Methotrexat-Intoxikation veröffentlicht. Es werden seither jedoch immer wieder Berichte mit irrtümlicher täglicher Einnahme gemeldet. Von 1997 bis Mitte 2015 wurden Swissmedic insgesamt 675 Berichte über unerwünschte Arzneimittelwirkungen (UAW) unter Methotrexat gemeldet. Davon:

- 18 akzidentelle Überdosierungen primär durch orale Einnahme, vereinzelt auch subkutane Gabe, täglich statt wöchentlich, wovon 4 tödlich ausgingen (2000, 2009 [2] und 2014).

Korrespondenz:

Dr. med. Beat Damke

Clinical Reviewer Swissmedic

beat.damke[at]swissmedic.ch www.swissmedicinfo.ch
- Bei 14 Meldungen erfolgte die tägliche Einnahme während mehr als 10 Tagen, bei 4 Meldungen während 6, 8, 8 und 9 Tagen

Die Fehler entstanden auf allen Ebenen an den Schnittstellen des Medikationsprozesses: Bei der ärztlichen Verordnung, der Verabreichung durch Pflegende oder Angehörige, der Abgabe in der Apotheke oder der Anwendung durch Patienten, durch fehlende oder mangelhafte Kommunikation. Sie beschränken sich nicht auf den Beginn der Therapie, sondern traten auch bei gut eingespielter Behandlung auf. Kritisch ist jeder Wechsel, z.B. von s.c. Fertigspritzen auf Tabletten, Wechsel der Institution, der Pflegenden.

Swissmedic stellt bei der Auswertung der gemeldeten Fälle auch fest, dass die Patienten häufig das Gefühl haben, die Einnahme einer Tablette nur $1 \times$ wöchentlich sei nicht ausreichend. Deshalb ist es umso wichtiger, dass die Patienten von den Fachpersonen ganz genau instruiert und überwacht werden.

Swissmedic klärt aktuell weitergehende Massnahmen ab, um akzidentelle Intoxikationen von Low Dose Methotrexat zu verhindern.

\section{Fazit}

Alle Fachleute im Gesundheitswesen sollen darauf achten, dass Patienten mit Rheumatoider Arthritis Low Dose Methotrexat nur $1 \times$ wöchentlich einnehmen. Alle involvierten Fachpersonen, Patienten und ihre Angehörigen und alle weiteren Beteiligten sind entsprechend zu instruieren und die Patienten zu überwachen.

Bei Anzeichen einer möglichen Überdosierung wie Mukositis/Stomatitis, Anämie, Leukopenie, Thrombozytopenie oder akutem Nierenversagen sollte unbedingt die Frequenz der Medikationseinnahme überprüft werden.

Swissmedic ist mit den betroffenen Firmen, Patientenund Pflegeorganisationen, Fachverbänden und Standesorganisationen in Bezug auf weitere Massnahmen in Kontakt.

1 Abegglen J, Frank O, Hochreutener MA, Stoller R. Akzidentelle Überdosierung von Methotrexat. Schweiz Ärztezeitung. 2012; 93(49):1818 und Pharmajournal. 2012;(25):7. 